membranes, and a useful description of procedures for their isolation and fractionation together with explanations of how these affect the nature and properties of the isolated material. The composition and functions of bacterial membranes are then discussed in some detail. The main emphasis, however, is on cell walls, particularly those of bacteria, though two fairly short chapters are included on the structure and biosynthesis of components of walls of yeasts and filamentous fungi.

Detailed attention is given to the structure and biosynthesis of the component polymers of walls. The interrelation and function of these components are discussed but some of the physical properties of walls and the surface properties of cells are less fully described. For example, the chapter on ultrastructure gives values for the thickness of the walls of several Gram positive bacteria but there is little reference to the evidence suggesting that the native hydrated walls are considerably thicker than those seen in fixed thin section. Brief reference is made to the ability of peptidoglycan to change in volume with ionic conditions but the effects of the presence of anionic polymers in the wall are not discussed, nor are the surface-charge properties of microorganisms. No mention is made, either, of the flocculation of yeast, useful though that property has been over the last few thousand years.

While a fuller coverage of surface properties would require some consideration of capsules and surface appendages, I think that it would not have been out of place in a book such as this and could have been of interest and value, particularly to the undergraduate or more general reader. These might also have benefited from a rather more explicit description of some of the background material. Thus the final chapter, dealing with the assembly and growth of bacterial walls, makes only very brief reference to present or past thinking on the relation between genome segregation and wall growth and cell division: it does not describe topics such as the surface layers in growth and cell division in stalked and budding bacteria which, though not yet well understood at the molecular level, might have helped the non-expert reader to gain a wider perspective.

Opinions may differ on what would constitute an ideal balance but there is no doubt that this is a most useful and valuable book. The authors have faced a formidable task in selecting from the vast amount of information that is now available and have produced a clearly written and beautifully presented book, containing a wealth of references to reviews and original papers. It should interest a wide range of readers.

A.R. Archibald is Professor of Microbiological Chemistry at the University of Newcastle upon Tyne.

\title{
Problems and solutions for phytoplankton
}

\section{Karl Banse}

The Physiological Ecology of Phytoplankton. Studies in Ecology, Vol.7. Edited by I. Morris. Pp.625. ISBN 0-632-00395-2/ 0-520-04308-1. (Blackwell Scientific/ University of California Press: 1981 .) £36, \$60.

MANY of us who work with phytoplankton indulge in speaking about "algae", "diatoms" or "phytoplankton", and in treating nutrient uptake or population growth in terms of the entire assemblage. This approach is in part forced upon us by our methods, for example the measurement of photosynthesis in a bottle of natural water. In part, however, we may be seduced by the apparent simplicity of a system of cells bathed individually by the environment and having high growth rates; they should be nearly in equilibrium with their surroundings. Investigators of larger, longer-lived invertebrates would hardly think of their objects of study in this way.

The last two chapters of the present volume, about succession and evolution, have reminded me about the individual phytoplankton species, of their problems of living, their solutions to these problems and of our ignorance. Further, in the other more conventional chapters I have been struck by the emergence and recurrent mention of studies of phase relations and time lags of responses in the laboratory, of the role of frequency of disturbance, and by the growing recognition of the importance of these phenomena in the field: algal cells are not necessarily in equilibrium with their surroundings but may lag behind the environmental change by a day or more.

The red thread running through this treatise is the interface between algal physiology and environmental factors. The subject has been tackled by commissioning in-depth, well-documented reviews which strive for a hydrobiological rather than a restricted marine scope. The first two chapters treat basic features of algal cells, symbioses and diseases, and general methodology. The latter subject resurfaces, often in great detail, in several of the subsequent seven contributions on photosynthesis, nutrient dynamics, trace metals (iron, manganese and copper) and vitamins. The chemistry chapters, strongly physiological in their orientation, are not uniformly organized but all review modern data of the distribution of the substances in natural waters. The last six discuss sinking and flotation, patchiness of distributions, the role of algal cell size, grazing, succession and evolution. Although these chapters were subsumed by the editor under the general heading of population dynamics, many concern themselves principally with effects on specific growth rates, as did the preceding chapters by their very nature.

The book, however, is much more than a collection of good to excellent but otherwise accidental reviews. The editor has achieved a broad coverage and, moreover, seems to have seen to it that there are appropriate cross-references between chapters. The large index is helpful but could have been better organized. For example, the subject of "biomass estimations" does not appear as such but as a subheading under the three species studied in this context, whereas the text referred to gives a general account of the subject. Likewise, "copper inhibition"' is not to be found under "copper" - so even today one can overdo the species bit!

In my opinion, this volume will be useful to the phytoplankton field worker (marine and freshwater), the advanced, general ecologist and the physiologist interested in ecological aspects of the subject. It is likely to be read or consulted for quite some time even though almost all manuscripts were apparently written in 1977 and the authors had little opportunity to add later references. The chapters are largely so specialized that the book as a whole cannot be used as a text; however, some contributions are to be recommended as a special reading for more senior students. $\square$

Karl Banse is Professor of Oceanography at the University of Washington, Seattle.

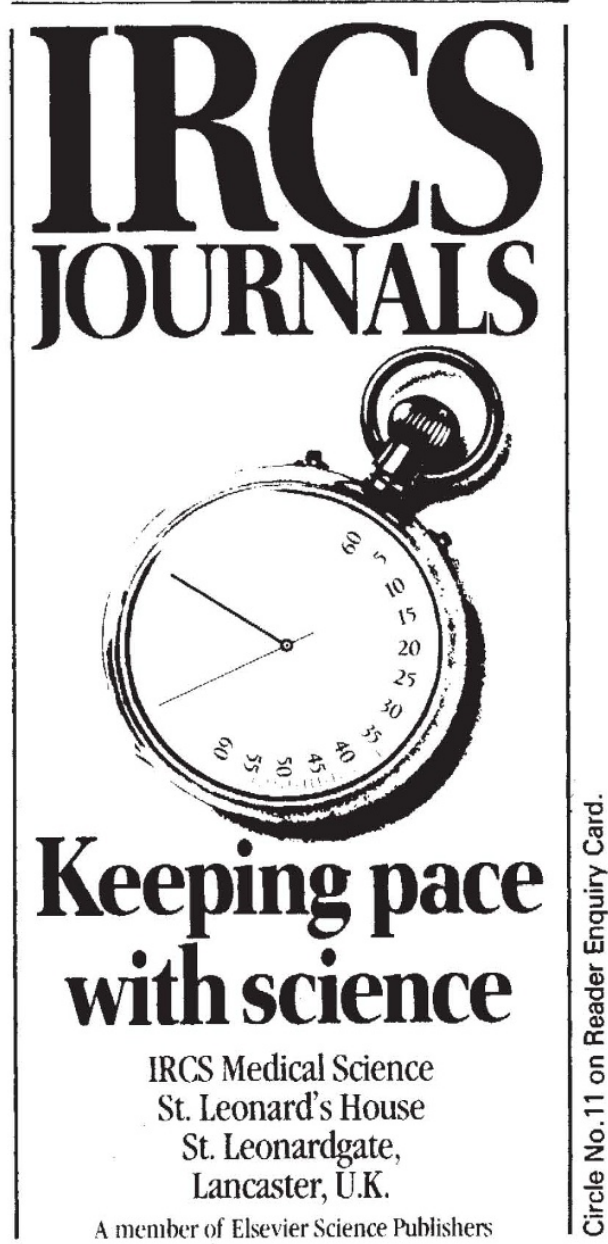

